\title{
Paris \\ Le prix Prud'homme 2001 remis à Françoise Vimeux
}

$\mathrm{U}$ ne fois n'est pas coutume : c'est dans les locaux spacieux et fonctionnels de la Maison de la chimie, dans $1 e 7^{\circ}$ arrondissement de Paris, que s'est déroulée la cérémonie de remise du prix André Prud'homme, le 19 décembre 2001. Un peu après 16 heures, une petite cinquantaine de personnes étaient rassemblées pour écouter les allocutions d'ouverture et la conférence de la lauréate.

Après avoir rappelé les principales activités de la SMF, Michel Petit, son président, a montré sous un jour nouveau l'intérêt du prix Prud'homme qui,

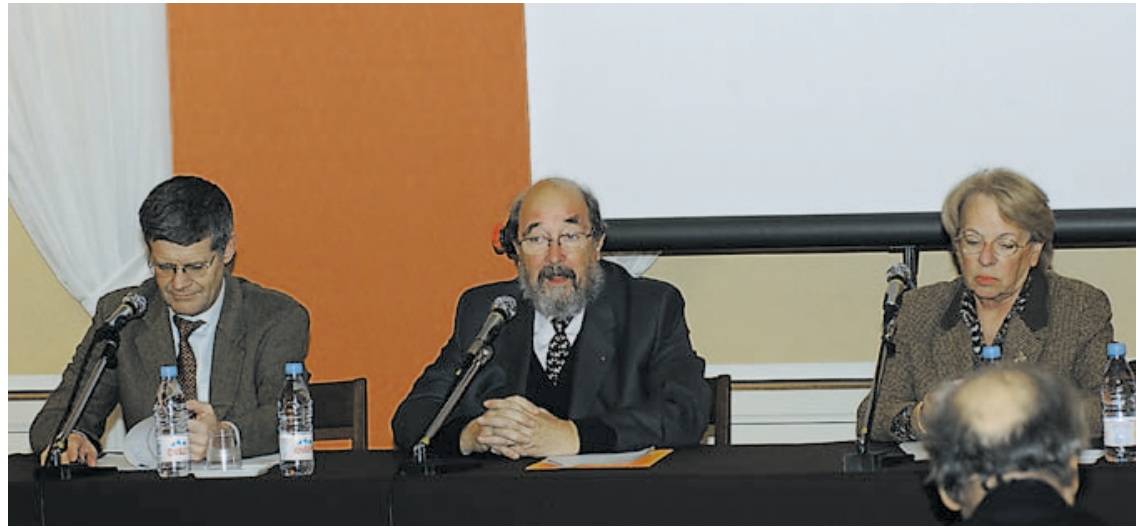

À la tribune pour les allocutions d'ouverture, de gauche à droite, Jean-Pierre Mac Veigh, Michel Petit et Marie-Lise Chanin. (Photos Météo-France, P. Taburet)

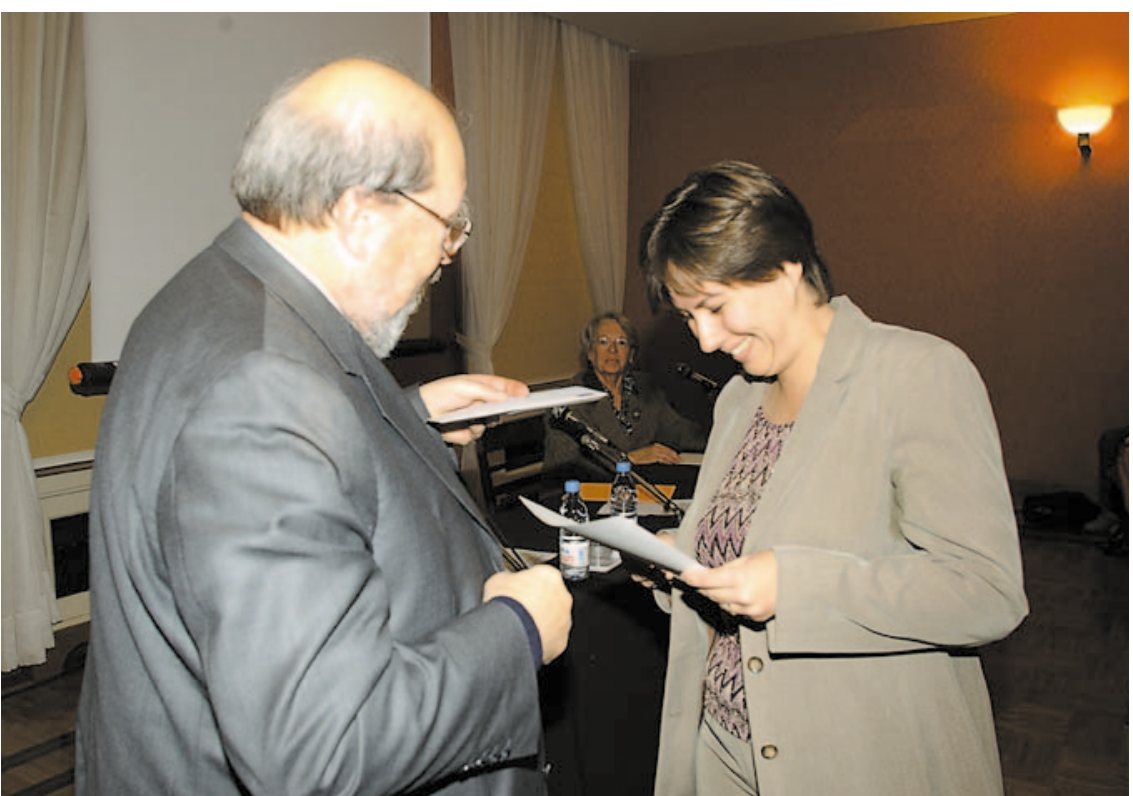

Michel Petit remet le prix Prud'homme 2001 à Françoise Vimeux.

rappelons-le, récompense la meilleure thèse de l'année en sciences de l'atmosphère et du climat. Selon lui, ce prix permet de mettre en lumière les travaux scientifiques de toute première importance et de compenser un certain laxisme de l'Université dans l'attribution de la mention « très honorable » aux thèses qu'elle décerne. De plus, le prix facilite grandement le début de carrière des thésards lauréats. JeanPierre Mac Veigh, qui représentait le président-directeur général de MétéoFrance, excusé, a ensuite réaffirmé l'attachement et le soutien du service météorologique français au prix Prud'homme. Enfin, Marie-Lise Chanin, représentant pour l'occasion le prési- dent du jury, Jean-Claude André, lui aussi excusé, a montré que le prix Prud'homme continuait à susciter un large intérêt de la part des jeunes chercheurs : pour le prix 2001, on a ainsi enregistré vingt-deux candidatures, provenant de douze laboratoires et représentant neuf universités plus Météo-France.

Le moment était venu pour Françoise Vimeux, la lauréate, de se voir remettre par Michel Petit le diplôme et le chèque de 10000 francs qui concrétisent le prix Prud'homme. Françoise Vimeux a mené les recherches en paléoclimatologie qui ont donné lieu

Dans l'assistance, on remarque la légitime fierté des parents et de la sœur de la lauréate. 

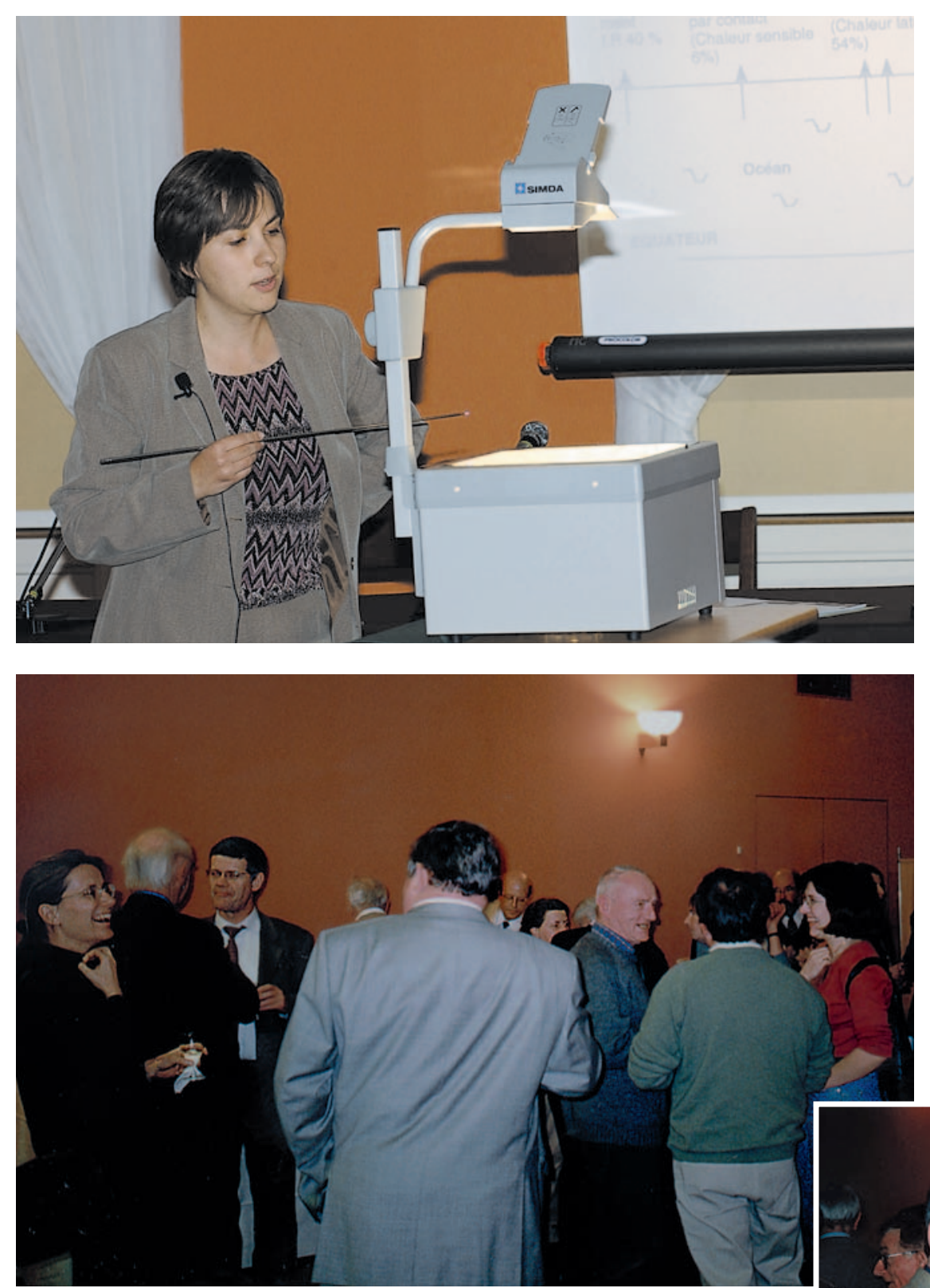

- Françoise Vimeux dans le difficile exercice de la vulgarisation.

- Cocktail sympathique et enjoué pour clore l'après-midi.

\section{Les lauréats successifs du prix Prud'homme}

\begin{tabular}{|c|c|c|}
\hline Année du prix & Lauréat & Discipline \\
\hline 1993 & Paolo Laj & Paléoclimatologie \\
\hline 1994 & Florence Rabier & Prévision numérique \\
\hline 1995 & Pascal Marquet & Météorologie dynamique \\
\hline 1996 & Olivier Boucher & Climatologie \\
\hline 1997 & Elsa Cortijo & Paléoclimatologie \\
\hline 1998 & Haraldur Ólafsson & Météorologie dynamique \\
\hline 1999 & Éric Guilyardi & Océanographie \\
\hline 2000 & $\begin{array}{l}\text { Philippe Arbogast } \\
\text { Fabio d'Andréa }\end{array}$ & $\begin{array}{l}\text { Météorologie dynamique } \\
\text { Météorologie dynamique }\end{array}$ \\
\hline 2001 & Françoise Vimeux & Paléoclimatologie \\
\hline
\end{tabular}

à sa thèse sous l'égide du Laboratoire des sciences du climat et de l'environnement (LSCE, laboratoire rattaché au CNRS et au CEA et situé à Saclay, dans l'Essonne) et sous la direction de Jean Jouzel.

Ce sont justement ces travaux de recherche que la lauréate a expliqués à l'assistance au cours de la conférence qui suivait. Les carottes de glace extraites du site de Vostok, en Antarctique, conservent des informations sur les précipitations qui sont tombées là-bas depuis 400000 ans (voir aussi l'article de V. MassonDelmotte et J. Chappellaz page 18 dans ce numéro de La Météorologie). Plus précisément, la teneur de cette eau archivée en isotopes stables (deutérium et oxygène-18 des molécules d'eau) permet de reconstituer certains aspects (température moyenne, intensité du cycle hydrologique, cycles glaciaireinterglaciaire...) de l'histoire climatique de notre planète.

Cela suppose, évidemment, un certain nombre d'hypothèses. Mais aussi un éventail encyclopédique de connaissances scientifiques, dans des domaines allant de la physico-chimie des isotopes à la théorie astronomique des climats, en passant par la modélisation numérique du climat. Françoise Vimeux a

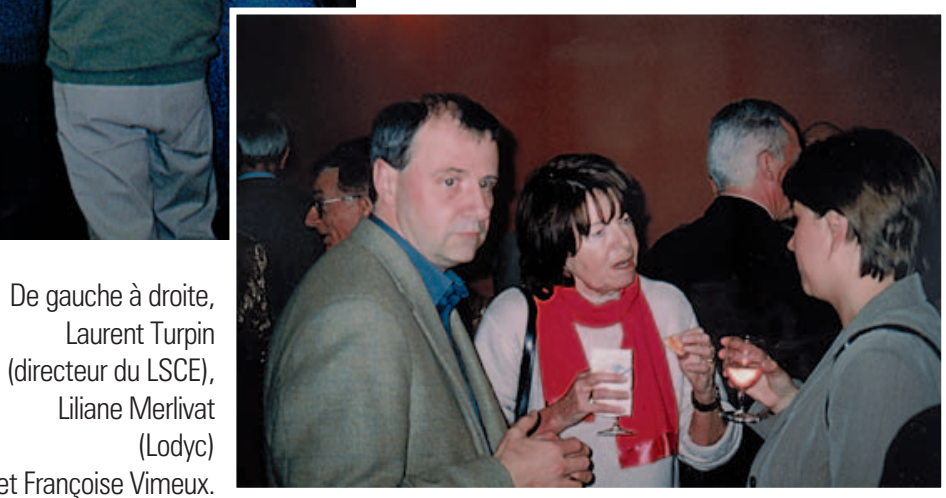

et Erancoise

brillamment démêlé cet écheveau et su mettre à la portée de son auditoire, avec simplicité et conviction, un sujet pourtant fort difficile.

Par ailleurs, la lauréate prépare actuellement un article de vulgarisation sur ce sujet, article destiné à paraître dans les colonnes de La Météorologie.

Didier Renaut

Météo-France et Société météorologique de France

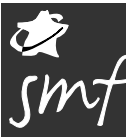

